\title{
Clinical outcomes of WBRT plus EGFR-TKIs versus WBRT or TKIs alone for the treatment of cerebral metastatic NSCLC patients: a meta-analysis
}

\author{
Hong Zheng ${ }^{1,}$, , Quan-Xing Liu ${ }^{1,},{ }^{*}$ Bin Hou ${ }^{1}$, Dong Zhou ${ }^{1}$, Jing-Meng Li ${ }^{1}$, Xiao Lu ${ }^{1}$, \\ Qiu-Ping $\mathbf{W u}^{1}$ and Ji-Gang Dai ${ }^{1}$ \\ ${ }^{1}$ Department of Thoracic Surgery, Xinqiao Hospital, Third Military Medical University, Chongqing 400037, China \\ *These authors contributed equally to this work
}

Correspondence to: Ji-Gang Dai, email: 691057831@qq.com

Qiu-Ping Wu, email: 2743389903@qq.com

Keywords: WBRT, EGFR-TKIS, NSCLC, BM

Received: April 19, 2017

Accepted: June 24, 2017

Published: July 06, 2017

Copyright: Zheng et al. This is an open-access article distributed under the terms of the Creative Commons Attribution License 3.0 (CC BY 3.0), which permits unrestricted use, distribution, and reproduction in any medium, provided the original author and source are credited.

\section{ABSTRACT}

Whether WBRT plus EGFR-TKIs has a greater survival benefit than EGFR-TKIs alone or WBRT alone remains controversial in NSCLC patients with multiple brain metastases. To rectify this, we conducted a systematic meta-analysis based on 9 retrospective studies and 1 randomized controlled study published between 2012 and 2016, comprising 1041 patients. Five studies were included in the comparison of WBRT plus EGFR-TKIs and EGFR-TKIs alone. The combined HR for OS of patients with EGFR mutation was 1.25 [95\% CI 0.98-2.15; $P=0.08]$ and for intracranial PFS was 1.30 [95\% CI 1.03-1.65; $P=0.03$ ], which revealed that EGFR-TKIs alone produced a superior intracranial PFS than WBRT plus EGFR-TKIs. Five studies were included in the comparison of WBRT plus EGFR-TKIs and WBRT alone. The combined HR for OS, intracranial PFS and extracranial PFS were $0.52[95 \%$ CI $0.37-0.75 ; P=0.0004], 0.36$ [95\% CI 0.24-0.53; $P<0.001]$ and 0.52 [95\% CI 0.38-0.71; $P<0.001]$, respectively, which revealed a significant benefit of WBRT plus EGFR-TKIs compared with WBRT alone. The results indicated that EGFR-TKIs alone should be the first option for the treatment of NSCLC patients with multiple BM, especially with EGFR mutation, since it provides similar OS and extracranial PFS but superior intracranial PFS compared with WBRT plus EGFR-TKIs.

\section{INTRODUCTION}

Brain metastasis in non-small cell lung cancer (NSCLC) is the most common intracranial metastatic tumor, and the incidence cases are more than half of all brain metastasis patients [1]. Approximately $25 \%$ to $40 \%$ of all NSCLC patients with brain metastases (BM) at some point in their disease course, and the risk is even higher in patients suffering from epidermal growth factor receptor (EGFR) mutation [2-4]. Furthermore, improved imaging in patients with prolonged survival due to the using of targeted systemic therapies in NSCLC with EGFR mutation is associated with a higher incidence of $\mathrm{BM}$ along with the disease process [5].
Therapeutic strategy for patients with BM vary according to the lesion number and size. Stereotactic radiosurgery (SRS) was always suitable for the patients with potentially superior prognosis [6]. However, whole brain radiation therapy (WBRT) has been regarded as the standard treatment for the tumor with a big size or those with $>3$ lesions, but commonly leads to neurologic sequelae, such as cognitive impairment. Most chemotherapeutic drugs are effectiveness in treatment of NSCLC with BM except brain radiotherapy. Though blood brain barrier (BBB) around brain metastasis is damaged, the concentration of chemotherapeutic drugs in brain is still not high enough, which is possibly caused by the pump-out of chemotherapeutic drugs by tumor cells through efflux 
pump. As a small molecule compound, EGFR-tyrosine kinase inhibitors (EGFR-TKIs) can penetrate through the $\mathrm{BBB}$ in certain proportions, becoming an important theoretic mechanism for the treatment of NSCLC patients with BM. A previously published review demonstrated the intracranial efficacy of EGFR-TKIs which was globally superior to the efficacy of standard chemotherapy [7].

In recent years, researchers have utilized EGFRTKIs to treat patients with brain metastasis [8-10]. Approaches adopted have included evaluating the role of EGFR-TKIs alone or with the concurrent use of EGFR-TKIs inhibition and WBRT in patients with brain metastases selected/unselected for EGFR mutation status. Several retrospective studies have compared the clinical outcomes of WBRT plus EGFR-TKIs and EGFRTKIs alone in NSCLC patients with BM. Seonggyu et al. reported that no significant differences were found between the WBRT plus EGFR-TKIs and the EGFR-TKIs alone regarding intracranial PFS, extracranial PFS and OS [11]. However, in another study by Qinghua Xu et al., they found that compared with WBRT alone and EGFR-TKIs alone, patients who received concomitant WBRT and EGFR-TKIs had the longest median survival time [12]. The discrepancy can be ascribed to the limited sample size of those studies. Meta-analysis was therefore urgently needed to systematically assess the quality of available evidence and make a scientific conclusion about WBRT plus EGFR-TKIs compared with EGFR-TKIs alone and WBRT alone in treating BM from NSCLC.

The purpose of the present study included two meta-analysis was to compare overall survival (OS) and intracranial/extracranial progression-free survival (PFS) outcomes of NSCLC patients with BM who received WBRT plus EGFR-TKIs, WBRT alone or EGFR-TKIs alone. Through the two pooled analysis, we hoped to form a consensus about whether WBRT plus EGFR-TKIs, WBRT alone or EGFR-TKIs alone is superior for NSCLC patients with BM.

\section{RESULTS}

\section{Characteristics of included trials}

A total of 861 records were identified from electronic databases and 10 references were tracked. Finally, five studies involving 598 patients were included in the study to compare clinical outcomes between WBRT plus EGFR-TKIs and EGFR-TKIs alone [11, 13-16]. In the meta-analysis to compare clinical outcomes between WBRT plus EGFR-TKIs and WBRT alone, 5 studies with 443 patients were included $[12,17-20]$. The search results and selection details are shown in Figure 1. Details for each study, including the, the status of EGFR mutation of primary lesion, the treatment procedure, the research year rang, the tumor stage for each trial and the publication year of the study were shown in Table 1 .

\section{WBRT plus EGFR-TKIs versus EGFR-TKIs alone}

After analyzed the practicable data in the existing researches, five studies [11, 13-16] involving 241 patients who received WBRT plus EGFR-TKIs were compared to 357 patients who received EGFR-TKIs alone to assess the overall survival from the date of treatment. As shown in Figure 2A, there was no significant difference in the OS $(\mathrm{HR}=1.00$ [95\% CI 0.81-1.24; $P=0.99])$. This result was coincident in the subgroup analysis which compared the OS between WBRT plus EGFR-TKIs and EGFRTKIs alone in EGFR mutation NSCLC patients with BM $(\mathrm{HR}=1.25$ [95\% CI 0.98-1.59; $P=0.08]$ ) (Figure 2B). Intracranial PFS and extracranial PFS were reported in two studies involving 110 patients who underwent WBRT plus EGFR-TKIs and 178 patients who underwent EGFR-TKIs alone. As shown in Figure $2 \mathrm{C}$, the meta-analysis revealed that compared with WBRT plus EGFR-TKIs for NSCLC patients with BM, the EGFR-TKIs alone treatment exhibited a superior intracranial PFS (HR $=1.30$ [95\% CI $1.03-1.65 ; P=0.03]$ ) (Figure $2 \mathrm{C}$ ). However, there was no significant difference in extracranial PFS, as the combined HR was 1.11 [95\% CI 0.87-1.42; $P=0.38$ ] (Figure 2D).

\section{WBRT plus EGFR-TKIs versus WBRT alone}

A total of five studies [12, 17-20] were included as data sources for the meta-analysis comparing clinical outcomes between WBRT plus EGFR-TKIs and WBRT alone. There were five studies involved in the analysis of the overall survival; 189 patients underwent WBRT plus EGFR-TKIs and 254 patients underwent WBRT alone. As shown in Figure 3A, the meta-analysis indicated that the OS for WBRT plus EGFR-TKIs was superior to WBRT alone in NSCLC patients with BM $(\mathrm{HR}=0.52[95 \% \mathrm{CI}$ $0.37-0.75 ; P=0.0004]$ ). Two studies were included in the pooled analysis of intracranial PFS, with 42 patients treated by WBRT plus EGFR-TKIs and 56 patients treated by WBRT alone. The combined HR was 0.36 [95\% CI 0.24-0.53; $P<0.00001]$ (Figure 3B). When comparing the extracranial PFS between WBRT plus EGFR-TKIs and WBRT alone, only two studies were included, with 88 patients treated by WBRT plus EGFR-TKIs and 123 patients treated by WBRT alone. The combined HR was 0.52 [95\% CI 0.38-0.71; $P<0.0001]$ (Figure 3C). These pooled analysis revealed that compared with WBRT alone, WBRT plus EGFR-TKIs has a significant benefit for NSCLC patients with BM.

\section{Sensitivity analysis and publication bias}

To determine whether individual studies unduly influenced overall results, the analyses were repeated, excluding each study one at a time; no significant discrepancies in the outcomes were identified. The results 
Table 1: Description of studies comparing WBRT+TKIs between TKIs or WBRT only

\begin{tabular}{|c|c|c|c|c|c|c|c|c|c|c|c|}
\hline Author & Year & $\begin{array}{c}\text { WBRT+TKIs } \\
\text { vs } \\
\text { TKIs }\end{array}$ & $\begin{array}{c}\text { WBRT+TKIs } \\
\text { vs } \\
\text { WBRT }\end{array}$ & $\begin{array}{l}\text { Research } \\
\text { year } \\
\text { range }\end{array}$ & $\begin{array}{c}\text { Follow up } \\
\text { (m) }\end{array}$ & $\begin{array}{l}\text { Tumor } \\
\text { stage }\end{array}$ & $\begin{array}{c}\text { Percentage } \\
\text { patients with } \\
\text { EGFR mutation } \\
\text { in primary lesion }\end{array}$ & WBRT & TKIs & $\begin{array}{c}\text { Research } \\
\text { type }\end{array}$ & $\begin{array}{l}\text { NOS } \\
\text { score }\end{array}$ \\
\hline Yin-Duo Zeng [15] & 2012 & $45 \mathrm{vs} 45$ & & 2005-2009 & $1-60$ & NA & $12 / 90$ & $40 \mathrm{~Gy} / 20 \mathrm{f} / 4 \mathrm{w}$ & $\begin{array}{l}\text { gefitinib }(250 \mathrm{mg}) \\
\text { per day }\end{array}$ & retrospective & 7 \\
\hline Yang-Si Li [14] & 2016 & $33 \mathrm{vs} 55$ & & 2011-2015 & $1-40$ & IV & $100 \%$ & $\begin{array}{l}30 \mathrm{~Gy} / 10 \text { day } \\
\text { fractions }\end{array}$ & $\begin{array}{c}\text { gefitinib/erlotinib/ } \\
\text { icotinib/afatinib and } \\
\text { sorafenib }\end{array}$ & retrospective & 8 \\
\hline Tao Jiang [13] & 2016 & 51 vs116 & & 2012-2015 & $1-40$ & IV(M1b) & $100 \%$ & $\begin{array}{l}30 \mathrm{~Gy} / 10 \text { day } \\
\text { fractions }\end{array}$ & $\begin{array}{c}\text { gefitinib }(250 \mathrm{mg}) \text { or } \\
\text { erlotinib }(150 \mathrm{mg}) \text { or } \\
\text { icotinib }(150 \mathrm{mg}) \text { per } \\
\text { day; oral delivery }\end{array}$ & retrospective & 7 \\
\hline $\begin{array}{l}\text { Seonggyu-Byeon } \\
{[11]}\end{array}$ & 2016 & $57 \mathrm{vs} 57$ & & 2005-2013 & $0.4-47.9$ & IIIB/IV & $100 \%$ & $\begin{array}{l}2000 \text { cGy for } \\
5 \text { days over a } \\
\text { single week }\end{array}$ & $\begin{array}{l}\text { gefitinib }(250 \mathrm{mg}) \text { or } \\
\text { erlotinib }(150 \mathrm{mg}) \\
\text { per day; oral delivery }\end{array}$ & retrospective & 6 \\
\hline $\begin{array}{l}\text { Yong-shun Chen } \\
\text { [16] }\end{array}$ & 2016 & 53 vs 79 & & 2008-2014 & $3-80$ & IV & $100 \%$ & $\begin{array}{c}30 \mathrm{~Gy} / 10 \mathrm{f} \text { for } 5 \\
\text { days per week, } \\
\text { up to } 2 \text { weeks }\end{array}$ & $\begin{array}{l}250 \mathrm{mg} \text { gefitinib or } \\
150 \mathrm{mg} \text { erlotinib }\end{array}$ & retrospective & 7 \\
\hline T Komatsu [19] & 2013 & & 19 vs 25 & 2005-2011 & $1-50$ & NA & $5 / 44$ & $\begin{array}{l}30 \text { Gy (range, } \\
24-50 \text { Gy) }\end{array}$ & $\begin{array}{l}\text { gefitinib }(250 \mathrm{mg}) \text { or } \\
\text { erlotinib }(150 \mathrm{mg})\end{array}$ & retrospective & 7 \\
\hline Y Cai [20] & 2013 & & $65 \mathrm{vs} 92$ & 2009-2012 & $1-26.6$ & NA & $43 / 157$ & $\begin{array}{c}29.37 \sim 41.24 \mathrm{~Gy}, \\
3 \mathrm{~Gy} / \mathrm{d}, 5 \mathrm{times} / \\
\text { week }\end{array}$ & $\begin{array}{l}\text { gefitinib }(250 \mathrm{mg}) \text { or } \\
\text { erlotinib }(150 \mathrm{mg})\end{array}$ & retrospective & 6 \\
\hline H Zhuang [17] & 2013 & & $23 v s 31$ & 2009-2011 & $1-30$ & I-IV & $11 / 54$ & $\begin{array}{c}30 \mathrm{~Gy} / 10 \mathrm{f}, \\
5 \text { days per week, } \\
\text { up to } 2 \text { weeks }\end{array}$ & $150 \mathrm{mg}$ erlotinib & retrospective & 6 \\
\hline SM Lee [18] & 2014 & & $40 v s 40$ & 2009-2010 & $1-15$ & NA & $1 / 35$ & $\begin{array}{c}20 \mathrm{~Gy} \text { in } \\
5 \text { daily fractions }\end{array}$ & $150 \mathrm{mg}$ erlotinib & randomized & \\
\hline $\mathrm{Q} \mathrm{Xu}$ [12] & 2015 & & $42 v s 66$ & 2006-2013 & $1-48$ & NA & $11 / 108$ & $30 \mathrm{~Gy} / 10 \mathrm{fx}$ & $\begin{array}{l}\text { gefitinib }(250 \mathrm{mg}) \text { or } \\
\text { erlotinib }(150 \mathrm{mg})\end{array}$ & retrospective & 8 \\
\hline
\end{tabular}

TKIs: epidermal growth factor receptor tyrosine kinase inhibitors; WBRT: whole brain radiotherapy; Gy: gray (absorbed dose); w: week; d: day; fx: fractions; NOS score: Newcastle-Ottawa Scale score; NA: not available.

were similar no matter random or fixed-effects models were performed. Publication bias was determined by asymmetry of the funnel plot which was used to estimate the precision of the trials (Figure 4A-4B). Each circle represents the treatment effect expressed as the logarithm of the HR of OS in each trial plotted against the standard error as a measure of study size. The perpendicular line exhibits the pooled estimate of the meta-analysis. Funnel plot analysis on the OS/PFS of comparisons did not indicate significant publication bias.

\section{DISCUSSION}

The improvement of the clinical outcomes for NSCLC patients was owing to the deepen understanding the underlying molecular mechanism of lung cancer [17]. Recently, EGFR-TKIs have been considered as the standard first-line therapy for the EGFR mutant NSCLC patients, due to a series of large randomized phase III clinical trials uniformly revealed that treatment of EGFRTKI achieved a higher response rate and longer PFS [18-20]. Although the low concentration ratios of brainto-plasma for EGFR-TKIs $(<1-3 \%)$ have been reported, several prospective studies have shown that the treatment of EGFR-TKIs has a positive activity in NSCLC patients with multiple BM and EGFR mutation, with a response rate of up to $80 \%$ [21-23]. The status of the EGFR mutation in previous studies was always determined through detecting primary lesions; however, there is no evidence that whether NSCLC patients with BM should be treated with TKIs depends on the EGFR mutation status of primary lesions. The most direct reason is that we were unable to acquire metastatic lesions.

WBRT has been considered as the cornerstone treatment for multiple BM of NSCLC patients for decades $[24,25]$. Currently, the efficacy of WBRT might be enhanced by EGFR TKI based on some reported evidence. Preclinical data reported by Zhuang $\mathrm{H}$ et al. and Chinnaiyan $P$ et al. suggested that EGFR TKI synergizes radiation therapy in tumor control $[26,27]$. According to some published clinical studies, compared with WBRT alone, EGFR TKI plus WBRT demonstrated an improved therapeutic effect in multiple BM of NSCLC patients $[28,29]$. In addition, some studies demonstrated that in NSCLC patients with EGFR mutation and multiple $\mathrm{BM}$, even EGFR-TKIs alone promised similar survival effects compared with WBRT plus EGFR-TKIs [11,13]. However, the combination of WBRT and TKI remains questionable, especially considering that some studies reported that WBRT not only did not prolong the OS but also shortened the PFS. Therefore, we performed this meta-analysis to combine applicable studies to further examine the published data on this topic. This is the first meta-analysis on the combined OS/PFS of WBRT plus 
EGFR-TKIs versus WBRT alone and EGFR-TKIs alone for NSCLC patients with multiple BM.

In the meta-analysis comparing OS between WBRT plus EGFR-TKIs and EGFR-TKIs alone, no significant difference was found. To identify whether the results were influenced by the EGFR mutant status, we performed a subgroup analysis. Depending on the results of subgroup analysis in EGFR mutant NSCLC patients with multiple $\mathrm{BM}$, we considered that EGFR-TKIs alone yielded a superior OS for WBRT plus EGFR-TKIs, but there were no significant statistic differences. Furthermore, compared with WBRT plus EGFR-TKIs, the EGFR-TKIs alone revealed a significant superiority for intracranial PFS. However, patients who underwent EGFR-TKIs alone did not exhibit any significant differences for extracranial PFS when compared to patients who underwent WBRT plus
EGFR-TKIs. In addition, according to the meta-analysis comparing the OS/intracranial PFS/extracranial PFS between WBRT plus EGFR-TKIs and WBRT alone, we noticed that WBRT plus EGFR-TKIs is associated with significantly better OS, intracranial PFS and extracranial PFS in NSCLC patients with multiple BM.

William J.M et al found that upfront radiotherapy yield better outcomes for brain metastases in EGFR mutant NSCLC patients, the median OS in patients receiving upfront WBRT was 30 months, while the median OS was 25 months in those undergoing upfront EGFRTKIs [30]. However, it is still unclear whether there are superior OS for upfront of WBRT followed by EGFRTKI compared with WBRT combined with EGFR-TKIs according to the current studies. A prospective, multiinstitutional randomized trial of upfront WBRT followed

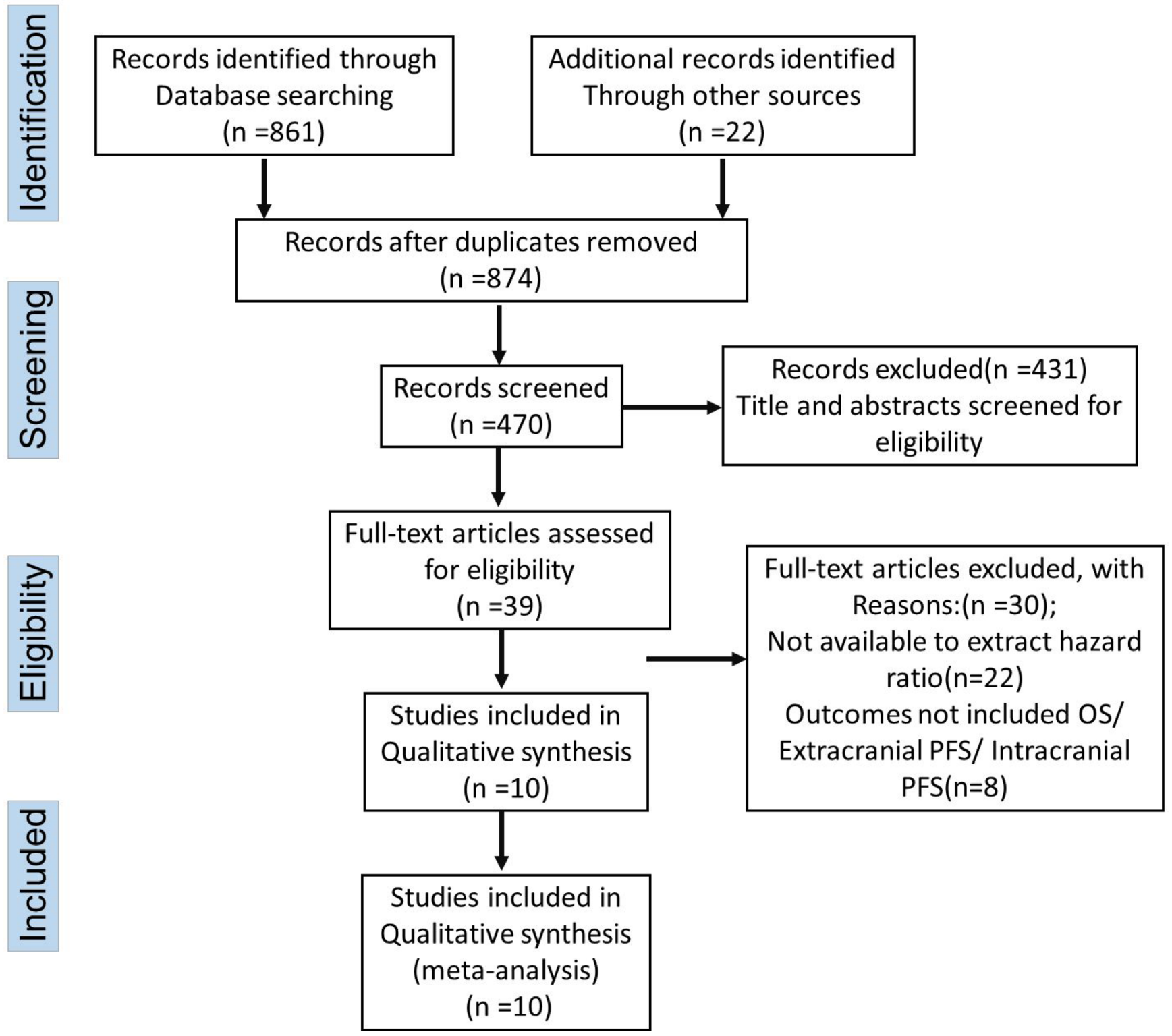

Figure 1: Flow chart of the literature retrieval according to the PRISMA statement. 
by EGFR-TKI versus WBRT combined with EGFR-TKI and TKI alone at iPFS and OS for NSCLC patients with multiple BM is urgently needed.

There are still several limitations in this study: (1) the level of evidence was relatively low, as most included articles were retrospective studies and whether clinical outcomes could be affected by undefined bias and/or confounding factors was unclear. (2) the radiation dose of WBRT and the type of EGFR-TKIs of the cohorts could not be collected and analyzed, which might have affected the survival of the NSCLC patients with multiple BM in some way. (3) we could not collect and analyse detailed the chemotherapy or surgery information of the cohorts, which might have influence on the outcomes of a few NSCLC patients in some respects. Despite the above limitations, the results of these two meta-analysis provided important evidence comparing the survival outcomes of WBRT plus EGFR-TKIs, EGFR-TKIs alone and WBRT alone in NSCLC patients with multiple BM.

In conclusion, these two meta-analysis revealed two important findings: (1) WBRT plus EGFR-TKIs produce significant superior OS, intracranial PFS and extracranial PFS to WBRT alone for NSCLC patients with multiple BM. (2) EGFR-TKIs alone produce similar OS and extracranial

A

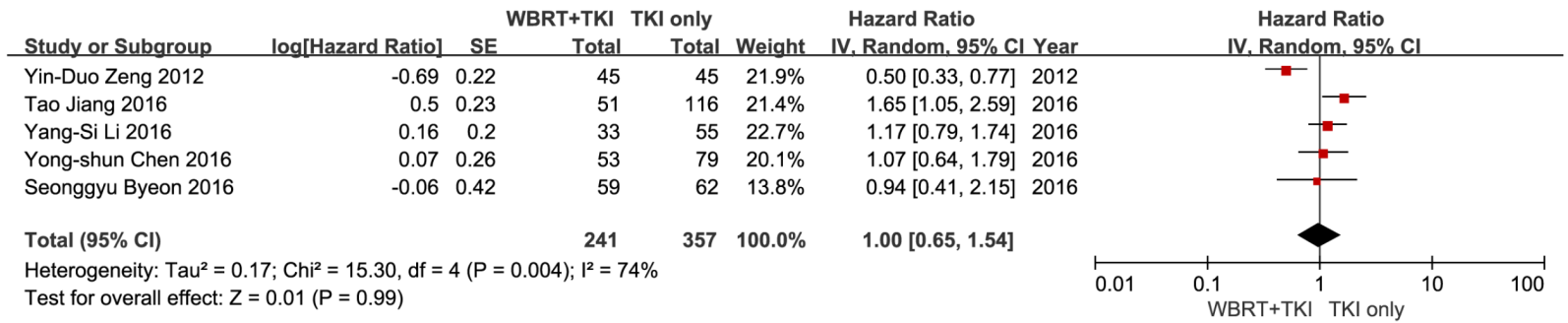

B

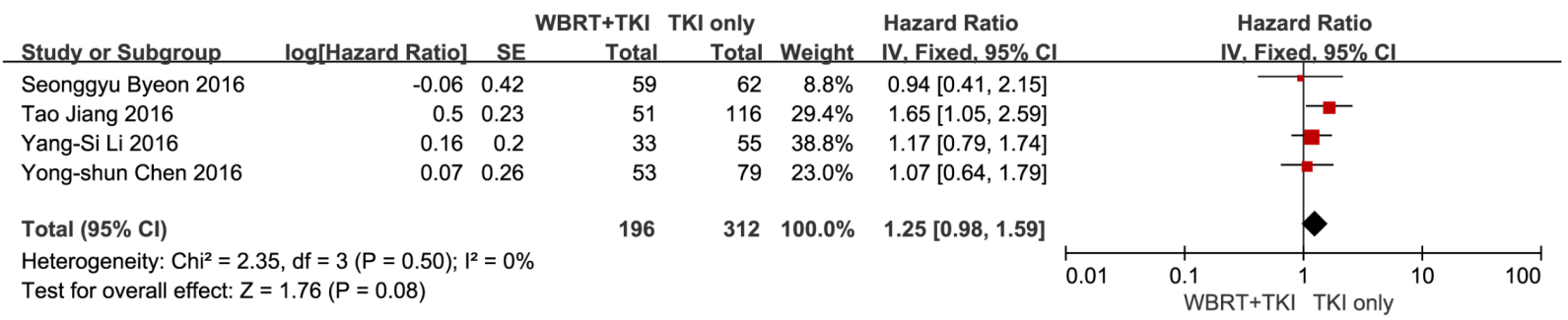

C

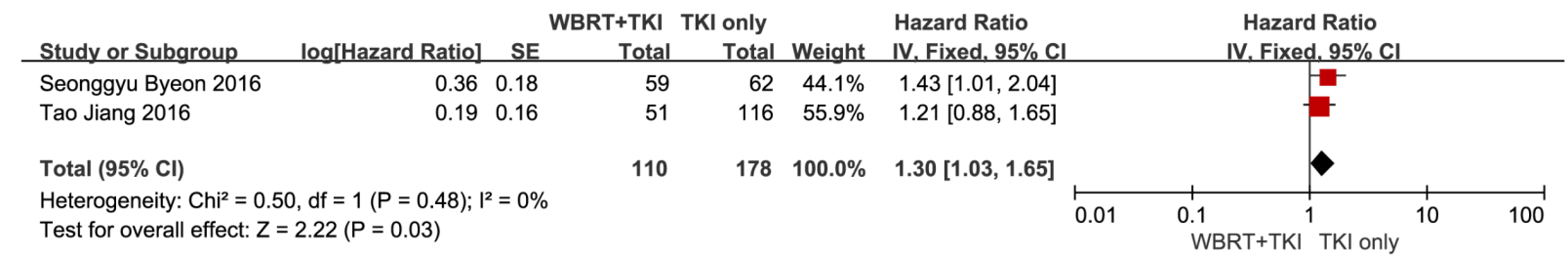

D

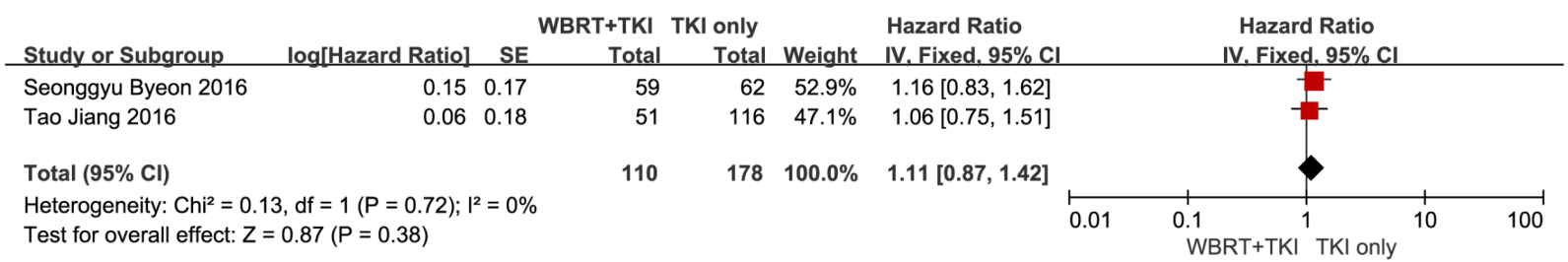

Figure 2: (A) Forest plot of comparison: the OS of WBRT+TKIs versus TKIs only in NSCLC patients with BM. Five studies were included. (B) Forest plot of comparison: the OS of WBRT+TKIs versus TKIs only in EGFR mutant NSCLC patients with BM. Four studies were included. (C) Forest plot of comparison: the intracranial PFS of WBRT+TKIs versus TKIs only in NSCLC patients with BM. Two studies were included. (D) Forest plot of comparison: the Extracranial PFS of WBRT+TKIs versus TKIs only in NSCLC patients with BM. Two studies were included. 
PFS but superior intracranial PFS with WBRT plus EGFRTKIs in NSCLC patients with multiple brain metastases.

Therefore, for the therapy of NSCLC patients with multiple BM, especially those with EGFR mutation, the first choice should be EGFR-TKIs alone when it is available.

\section{MATERIALS AND METHODS}

This study was designed according to the PRISMA statement [31]. To effectively identify included articles in our meta-analysis, a structured literature retrieval protocol

\section{A}

\begin{tabular}{|c|c|c|c|c|c|c|c|c|c|c|c|}
\hline Study or Subgroup & log[Hazard Ratio] & SE & $\begin{array}{c}\text { WBRT+TKI WBR } \\
\text { Total }\end{array}$ & $\begin{array}{l}\text { only } \\
\text { Total }\end{array}$ & & $\begin{array}{c}\text { Hazard Ratio } \\
\text { IV. Random, } 95 \% \mathrm{Cl}\end{array}$ & Year & & $\begin{array}{r}\text { Hazarc } \\
\text { IV. Rando }\end{array}$ & $\begin{array}{l}\text { d Ratio } \\
\text { om. } 95 \% \mathrm{Cl}\end{array}$ & \\
\hline H Zhuang 2013 & -0.92 & 0.41 & 23 & 31 & $12.0 \%$ & $0.40[0.18,0.89]$ & 2013 & & & & \\
\hline T Komatsu 2013 & -0.94 & 0.24 & 19 & 25 & $20.3 \%$ & $0.39[0.24,0.63]$ & 2013 & & & & \\
\hline Y Cai 2013 & -0.43 & 0.17 & 65 & 92 & $24.6 \%$ & $0.65[0.47,0.91]$ & 2013 & & & & \\
\hline SM Lee 2014 & -0.06 & 0.25 & 40 & 40 & $19.7 \%$ & $0.94[0.58,1.54]$ & 2014 & & & & \\
\hline Q Xu 2014 & -0.97 & 0.19 & 42 & 66 & $23.4 \%$ & $0.38[0.26,0.55]$ & 2014 & & & & \\
\hline Total $(95 \% \mathrm{Cl})$ & & & 189 & 254 & $100.0 \%$ & $0.52[0.37,0.75]$ & & & & & \\
\hline \multicolumn{4}{|c|}{$\begin{array}{l}\text { Heterogeneity: } \text { Tau }^{2}=0.10 ; \mathrm{Chi}^{2}=11.95, \mathrm{df}=4(P=0.02) ; \mathrm{I}^{2}=67 \% \\
\text { Test for overall effect: } Z=3.57(P=0.0004)\end{array}$} & & & & & 0.01 & $\begin{array}{l}0.1 \\
\text { WBRT+TKI }\end{array}$ & ${ }^{1} \quad 10$ & 100 \\
\hline
\end{tabular}

B

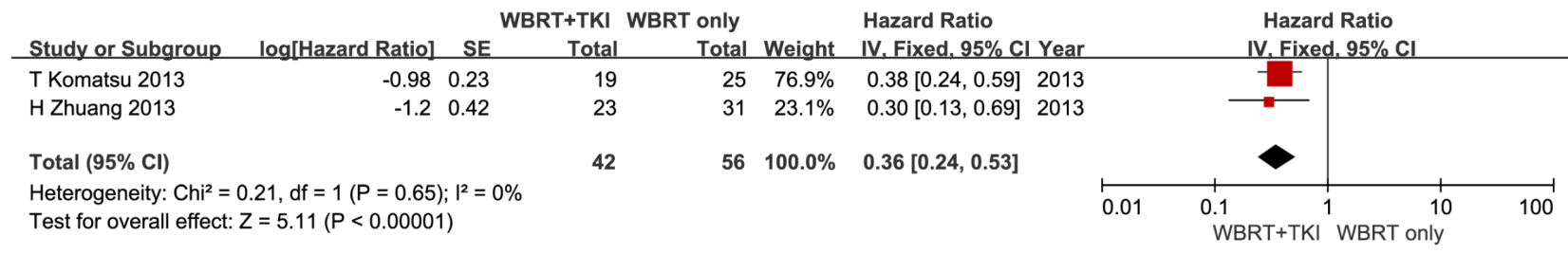

\section{C}

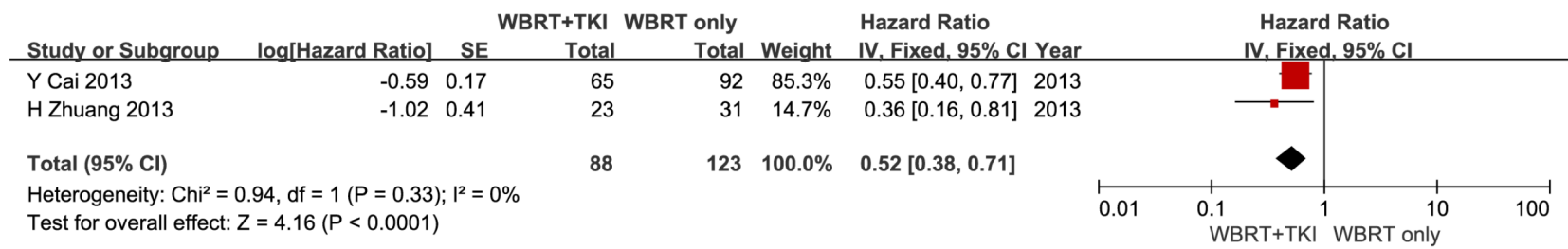

Figure 3: (A) Forest plot of comparison: the OS of WBRT+TKIs versus WBRT only in NSCLC patients with BM. Five studies were included. (B) Forest plot of comparison: the intracranial PFS of WBRT+TKIs versus WBRT only in NSCLC patients with BM. Two studies were included. (C) Forest plot of comparison: the Extracranial PFS of WBRT+TKIs versus WBRT only in NSCLC patients with BM. Two studies were included.

A

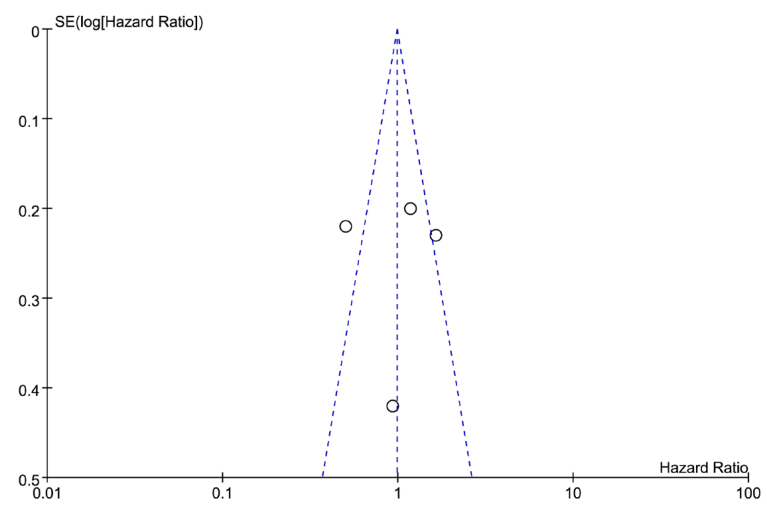

B

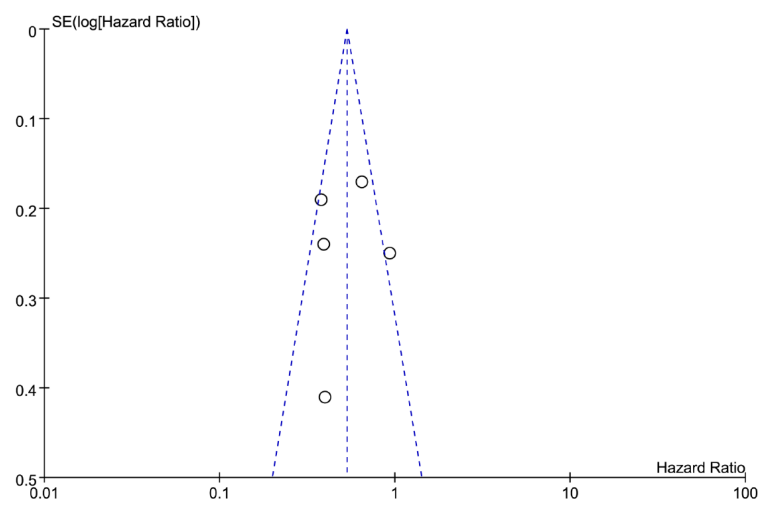

Figure 4: (A) Funnel plot of OS on the outcomes of the comparisons of WBRT+TKIs versus TKIs only in NSCLC patients with BM for the visual detection of systematic publication bias and small study effect. (B) Funnel plot of OS on the outcomes of the comparisons of WBRT+TKIs versus WBRT only in NSCLC patients with BM for the visual detection of systematic publication bias and small study effect. 
was formulated according to the recommendations from the Cochrane Collaboration. All the objective, inclusion and exclusion criteria, primary and secondary outcomes, and the methods of synthesis were pre-specified before the analyses.

\section{Search strategy for published studies}

We accomplished a literature retrieval for original researches by searching multiple electronic databases, including PubMed, MEDLINE, Embase, and ISI Web of Science, from their dates of inception to May 2017 using the following keywords related to lung cancer, WBRT, EGFR-TKIs, brain metastases and radiotherapy. The search and identification process was independently conducted by two authors (Hong Zheng and Quan Xing Liu). According to a standardized approach, and the selection of each study was reached by discussion. For purpose of acquiring the maximum sensitivity, we implemented the searching strategy as: "lung cancer" [all fields] AND "brain metastases" [all fields] AND "WBRT, radiotherapy" [MeSH Terms] OR "TKI" [all fields] OR “Gefitinib, Icotinib, Erlotinib" [all fields]. All the researches were filtered according to inclusion and exclusion criteria.

\section{Selection criteria}

Researches accorded with the following inclusion criteria were considered eligible and included in this metaanalysis: (1) all the patients included had NSCLC with multiple BM (>3); (2) study designs were prospective cohort studies or case-control studies; (3) provided data on OS or PFS; (4) intervention: WBRT plus EGFRTKIs alone and control: EGFR-TKIs or WBRT alone. Researches were excluded depended on the reasons listed below: (1) reviews, commentaries, editorials, case reports, and letters; (2) lacked key information for the calculation of methods; (3) published duplicate studies with the accumulating numbers of patients or increased lengths of follow-up were deliberated, only the most informative article was included in this meta-analysis.

\section{Quality assessment}

Quality of the included studies were assessed according to the Newcastle-Ottawa Scale (NOS) for the quality of cohort and case-control studies [32]. A star system for the NOS (range, 0-9 stars) was developed for the evaluation. The values for the included studies are shown in Table 1.

\section{Data extraction and critical appraisal}

OS and PFS were the primary outcomes. All data for analyzing were extracted from included eligible articles (all available texts, tables and figures). Each retrieved article was reviewed by two independent investigators. A third expert adjudicator and discussion were performed if there were discrepancies between the two reviewers.

\section{Statistical analysis}

The meta-analysis was performed by combining the results of the reported OS or PFS. The log (hazard ratio) $[\ln (\mathrm{HR})]$ and its standard error (SE) were used as the outcome measure for the combined data. Hazard ratio (HR) and associated variance data in each included study were obtained or calculated according to the techniques described by Tierney and Stewart [33]. Because the HR of OS/PFS could not obtained in some studies directly, data were extracted from the Kaplan-Meier survival curves of these studies to calculate the HR and SE of OS/DFS/CSS. Kaplan-Meier curves were read by Engauge Digitizer version 4.1 [34]. The calculations were performed independently by two researchers, and discrepancies were discussed to reach a consensus. The summary statistical analysis was conducted with Review Manager Version 5.1.2 (Cochrane Collaboration, Software Update, Oxford, United Kingdom). The heterogeneity between trials was assessed by using the Chi-square statistic; $\mathrm{I}^{2}$ less than $50 \%$ and a $P$ value greater than 0.10 suggested that there was no statistical heterogeneity. The inverse variance fixed effects model was applied for meta-analysis. And the inverse variance random effects model was used when clinical characteristics and methodology did not show great differences, $\mathrm{I}^{2}$ was greater than $50 \%$ and the $P$ value was less than 0.10 . The influence of the study regarding overall effect size was identified by sensitivity analysis.

\section{Authors' contributions}

1, Hong Zheng and Quan-Xing Liu are co-first authors, and they wrote the main manuscript text and prepared Figures 1-4. Qiu-ping $\mathrm{Wu}$ and Ji-Gang Dai contributed substantially to the idea and the design of analysis, and they gave the approval of the final version for publishing. 3, Dong Zhou, Jing-Meng Li and Xiao $\mathrm{Lu}$ accomplished the analysis and the interpretation of all data. 4, Ji-Gang Dai, Qiu-ping Wu and Bin Hou critically revised the article for important intellectual content.

\section{ACKNOWLEDGMENTS AND FUNDING}

The study was supported by the National Natural Science Foundation of China for Dai, No.81472188.

\section{CONFLICTS OF INTEREST}

The authors declare that there is no conflicts of interest. 


\section{REFERENCES}

1. Sperduto PW, Chao ST, Sneed PK, Luo X, Suh J, Roberge D, Bhatt A, Jensen AW, Brown PD, Shih H, Kirkpatrick J, Schwer A, Gaspar LE, et al. Diagnosis-specific prognostic factors, indexes, and treatment outcomes for patients with newly diagnosed brain metastases: a multi-institutional analysis of 4,259 patients. Int J Radiat Oncol Biol Phys. 2010; 77:655-661.

2. Zhang J, Yu J, Sun X, Meng X. Epidermal growth factor receptor tyrosine kinase inhibitors in the treatment of central nerve system metastases from non-small cell lung cancer. Cancer Lett. 2014; 351:6-12.

3. Costa R, Carneiro BA, Wainwright DA, Santa-Maria CA, Kumthekar P, Chae YK, Gradishar WJ, Cristofanilli M, Giles FJ. Developmental therapeutics for patients with breast cancer and central nervous system metastasis: current landscape and future perspectives. Ann Oncol. 2016.

4. Iuchi T, Shingyoji M, Itakura M, Yokoi S, Moriya Y, Tamura H, Yoshida Y, Ashinuma H, Kawasaki K, Hasegawa Y, Sakaida T, Iizasa T. Frequency of brain metastases in non-small-cell lung cancer, and their association with epidermal growth factor receptor mutations. Int J Clin Oncol. 2015; 20:674-679.

5. Eichler AF, Chung E, Kodack DP, Loeffler JS, Fukumura D, Jain RK. The biology of brain metastases-translation to new therapies. Nat Rev Clin Oncol. 2011; 8:344-356.

6. Tsao MN, Rades D, Wirth A, Lo SS, Danielson BL, Gaspar LE, Sperduto PW, Vogelbaum MA, Radawski JD, Wang JZ, Gillin MT, Mohideen N, Hahn CA, et al. Radiotherapeutic and surgical management for newly diagnosed brain metastasis(es): An American Society for Radiation Oncology evidence-based guideline. Pract Radiat Oncol. 2012; 2:210-225.

7. Barlesi F, Spano JP, Cortot AB, Carpentier AF, Robinet G, Besse $B$. [Systemic treatment of brain metastases from lung cancer]. [Article in French]. Cancer Radiother. 2015; 19:43-47.

8. Porta R, Sanchez-Torres JM, Paz-Ares L, Massuti B, Reguart N, Mayo C, Lianes P, Queralt C, Guillem V, Salinas P, Catot S, Isla D, Pradas A, et al. Brain metastases from lung cancer responding to erlotinib: the importance of EGFR mutation. Eur Respir J. 2011; 37:624-631.

9. Ceresoli GL, Cappuzzo F, Gregorc V, Bartolini S, Crino L, Villa E. Gefitinib in patients with brain metastases from non-small-cell lung cancer: a prospective trial. Ann Oncol. 2004; 15:1042-1047.

10. Iuchi T, Shingyoji M, Sakaida T, Hatano K, Nagano O, Itakura M, Kageyama $\mathrm{H}$, Yokoi S, Hasegawa Y, Kawasaki K, Iizasa T. Phase II trial of gefitinib alone without radiation therapy for Japanese patients with brain metastases from EGFR-mutant lung adenocarcinoma. Lung Cancer. 2013; 82:282-287.

11. Byeon S, Ham JS, Sun JM, Lee SH, Ahn JS, Park K, Ahn MJ. Analysis of the benefit of sequential cranial radiotherapy in patients with EGFR mutant non-small cell lung cancer and brain metastasis. Med Oncol. 2016; 33:97.

12. Xu Q, Chen X, Qian D, Wang Y, Meng S, Liu H, Zhou C. Treatment and prognostic analysis of patients with leptomeningeal metastases from non-small cell lung cancer. Thorac Cancer. 2015; 6:407-412.

13. Jiang T, Su C, Li X, Zhao C, Zhou F, Ren S, Zhou C, Zhang J. EGFR TKIs plus WBRT Demonstrated No Survival Benefit Other Than That of TKIs Alone in Patients with NSCLC and EGFR Mutation and Brain Metastases. J Thorac Oncol. 2016; 11:1718-1728.

14. Li YS, Jiang BY, Yang JJ, Tu HY, Zhou Q, Guo WB, Yan HH, Wu YL. Leptomeningeal Metastases in Patients with NSCLC with EGFR Mutations. J Thorac Oncol. 2016; 11:1962-1969.

15. Zeng YD, Zhang L, Liao H, Liang Y, Xu F, Liu JL, Dinglin XX, Chen LK. Gefitinib alone or with concomitant whole brain radiotherapy for patients with brain metastasis from non-small-cell lung cancer: a retrospective study. Asian Pac J Cancer Prev. 2012; 13:909-914.

16. Chen Y, Yang J, Li X, Hao D, Wu X, Yang Y, He C, Wang W, Wang J. First-line epidermal growth factor receptor (EGFR)-tyrosine kinase inhibitor alone or with whole-brain radiotherapy for brain metastases in patients with EGFR-mutated lung adenocarcinoma. Cancer Sci. 2016; 107:1800-1805.

17. Mok T, Yang JJ, Lam KC. Treating patients with EGFRsensitizing mutations: first line or second line--is there a difference? J Clin Oncol. 2013; 31:1081-1088.

18. Mok TS, Wu YL, Thongprasert S, Yang CH, Chu DT, Saijo N, Sunpaweravong P, Han B, Margono B, Ichinose Y, Nishiwaki Y, Ohe Y, Yang JJ, et al. Gefitinib or carboplatinpaclitaxel in pulmonary adenocarcinoma. N Engl J Med. 2009; 361:947-957.

19. Zhou $\mathrm{C}$, Wu YL, Chen G, Feng J, Liu XQ, Wang C, Zhang S, Wang J, Zhou S, Ren S, Lu S, Zhang L, Hu C, et al. Erlotinib versus chemotherapy as first-line treatment for patients with advanced EGFR mutation-positive nonsmall-cell lung cancer (OPTIMAL, CTONG-0802): a multicentre, open-label, randomised, phase 3 study. Lancet Oncol. 2011; 12:735-742.

20. Wu YL, Zhou C, Hu CP, Feng J, Lu S, Huang Y, Li W, Hou M, Shi JH, Lee KY, Xu CR, Massey D, Kim M, et al. Afatinib versus cisplatin plus gemcitabine for first-line treatment of Asian patients with advanced non-small-cell lung cancer harbouring EGFR mutations (LUX-Lung 6): an open-label, randomised phase 3 trial. Lancet Oncol. 2014; 15:213-222.

21. Park SJ, Kim HT, Lee DH, Kim KP, Kim SW, Suh C, Lee JS. Efficacy of epidermal growth factor receptor tyrosine kinase inhibitors for brain metastasis in non-small cell lung cancer patients harboring either exon 19 or 21 mutation. Lung Cancer. 2012; 77:556-560.

22. Iuchi T, Shingyoji M, Sakaida T, Hatano K, Nagano O, Itakura M, Kageyama $\mathrm{H}$, Yokoi S, Hasegawa Y, 
Kawasaki K, Iizasa T. Phase II trial of gefitinib alone without radiation therapy for Japanese patients with brain metastases from EGFR-mutant lung adenocarcinoma. Lung Cancer. 2013; 82:282-287.

23. Welsh JW, Komaki R, Amini A, Munsell MF, Unger W, Allen PK, Chang JY, Wefel JS, McGovern SL, Garland LL, Chen SS, Holt J, Liao Z, et al. Phase II trial of erlotinib plus concurrent whole-brain radiation therapy for patients with brain metastases from non-small-cell lung cancer. J Clin Oncol. 2013; 31:895-902.

24. Reveiz L, Rueda JR, Cardona AF. Chemotherapy for brain metastases from small cell lung cancer. Cochrane Database Syst Rev. 2012; :CD007464.

25. Li J, Bentzen SM, Renschler M, Mehta MP. Regression after whole-brain radiation therapy for brain metastases correlates with survival and improved neurocognitive function. J Clin Oncol. 2007; 25:1260-1266.

26. Zhuang H, Wang J, Zhao L, Yuan Z, Wang P. The theoretical foundation and research progress for WBRT combined with erlotinib for the treatment of multiple brain metastases in patients with lung adenocarcinoma. Int J Cancer. 2013; 133:2277-2283.

27. Chinnaiyan P, Huang S, Vallabhaneni G, Armstrong E, Varambally S, Tomlins SA, Chinnaiyan AM, Harari PM. Mechanisms of enhanced radiation response following epidermal growth factor receptor signaling inhibition by erlotinib (Tarceva). Cancer Res. 2005; 65:3328-3335.

28. Gow CH, Chien CR, Chang YL, Chiu YH, Kuo SH, Shih JY, Chang YC, Yu CJ, Yang CH, Yang PC. Radiotherapy in lung adenocarcinoma with brain metastases: effects of activating epidermal growth factor receptor mutations on clinical response. Clin Cancer Res. 2008; 14:162-168.
29. Soon YY, Leong CN, Koh WY, Tham IW. EGFR tyrosine kinase inhibitors versus cranial radiation therapy for EGFR mutant non-small cell lung cancer with brain metastases: a systematic review and meta-analysis. Radiother Oncol. $2015 ; 114: 167-172$.

30. Magnuson WJ, Lester-Coll NH, Wu AJ, Yang TJ, Lockney NA, Gerber NK, Beal K, Amini A, Patil T, Kavanagh BD, Camidge DR, Braunstein SE, Boreta LC, et al. Management of Brain Metastases in Tyrosine Kinase Inhibitor-Naive Epidermal Growth Factor Receptor-Mutant Non-Small-Cell Lung Cancer: A Retrospective Multi-Institutional Analysis. J Clin Oncol. 2017; 35:1070-1077.

31. Liberati A, Altman DG, Tetzlaff J, Mulrow C, Gotzsche PC, Ioannidis JP, Clarke M, Devereaux PJ, Kleijnen J, Moher D. The PRISMA statement for reporting systematic reviews and meta-analyses of studies that evaluate healthcare interventions: explanation and elaboration. BMJ. 2009; 339:b2700.

32. Chen C, Xu T, Chen J, Zhou J, Yan Y, Lu Y, Wu S. Allergy and risk of glioma: a meta-analysis. Eur J Neurol. 2011; 18:387-395.

33. Tierney JF, Stewart LA, Ghersi D, Burdett S, Sydes MR. Practical methods for incorporating summary time-to-event data into meta-analysis. Trials. 2007; 8:16.

34. Cao C, Gupta S, Chandrakumar D, Tian DH, Black D, Yan TD. Meta-analysis of intentional sublobar resections versus lobectomy for early stage non-small cell lung cancer. Ann Cardiothorac Surg. 2014; 3:134-141. 